\title{
Is breastfeeding protective against the development of asthma or wheezing in children? A systematic review and meta-analysis
}

\author{
Elinor Simons ${ }^{1 *}$, Sharon D Dell ${ }^{1,2}$, Joseph Beyene ${ }^{3,4}$, Teresa To ${ }^{1}$, Prakesh S Shah ${ }^{5}$ \\ From Canadian Society of Allergy and Clinical Immunology Annual Scientific Meeting 2011 \\ Quebec, Canada. 20-23 October 2011
}

\section{Background}

Although breastfeeding is strongly recommended for its many benefits, the association between breastfeeding and childhood asthma development remains controversial. Our objective was to systematically review and metaanalyze the association between physician-diagnosed asthma (PDA) or wheezing development and exclusive or any breastfeeding.

\section{Methods}

Prospective cohort studies of preschool (4-6 years) and school-aged (7-9 years) children were identified from Medline (1948-June 2011) and Embase (1980-June 2011). Breastfeeding exposure for at least the first 3-4 months of life was defined as exclusive (breast milk as the only source of nutrition) or any (breast milk included in the diet). Outcomes were parent-reported PDA or wheezing. Risk of bias in included studies was assessed using the Newcastle-Ottawa scale. Data were analyzed using the Revman software package and adjusted odds ratios were meta-analyzed using random-effects models.

\section{Results}

Ten studies enrolling 35,411 participants were included. Decreased odds of PDA or wheezing development at ages 7-9 years were identified for those who received exclusive breastfeeding [adjusted odds ratio (OR) 0.69, 95\% confidence interval (CI): 0.58-0.83] and any breastfeeding (OR 0.53, 95\% CI: 0.41-0.68) and at ages 4-6 years for those who received exclusive breastfeeding (OR 0.75, 95\% CI: 0.61-0.93). Among the clinically-heterogeneous studies with outcome assessment at ages 4-6 years, any breastfeeding did not change the odds of PDA or wheezing (OR 1.08, 95\% CI: 0.76-1.54).

\section{Conclusions}

Exclusive or any breastfeeding for at least the first 3-4 months of life was associated with lower odds of PDA or wheezing in children, strengthening support for the current breastfeeding recommendations.

\section{Author details}

${ }^{1}$ Child Health Evaluative Sciences, Hospital for Sick Children, Toronto, ON, Canada, M5G 1 X8. ${ }^{2}$ Division of Respiratory Medicine, Hospital for Sick Children, Toronto, ON, Canada, M5G 1X8. ${ }^{3}$ Population Health Sciences, Hospital for Sick Children, Toronto, ON, Canada, M5G 1X8. ${ }^{4}$ Clinical Epidemiology and Biostatistics, McMaster University, Hamilton, ON, Canada, L8S 4K1. ${ }^{5}$ Division of Neonatology, Mount Sinai Hospital, Toronto, ON, Canada, M5G $1 \times 5$.

Published: 14 November 2011

doi:10.1186/1710-1492-7-S2-A11

Cite this article as: Simons et al.: Is breastfeeding protective against the development of asthma or wheezing in children? A systematic review and meta-analysis. Allergy, Asthma \& Clinical Immunology 2011 7(Suppl 2): A11.

\footnotetext{
* Correspondence: elinor.simons@sickkids.ca

${ }^{1}$ Child Health Evaluative Sciences, Hospital for Sick Children, Toronto, ON,

Canada, M5G 1 X8

Full list of author information is available at the end of the article
}

(c) 2011 Simons et al; licensee BioMed Central Ltd. This is an open access article distributed under the terms of the Creative Commons 